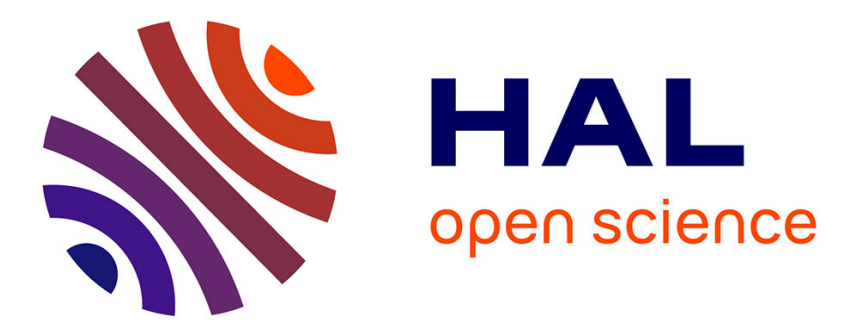

\title{
Development of a set of SNP markers for population genetics of the red gorgonian (Paramuricea clavata), an emblematic species of the Mediterranean coralligenous
}

M. Padrón, M. Milhes, M. Massot, E. Guichoux

\section{- To cite this version:}

M. Padrón, M. Milhes, M. Massot, E. Guichoux. Development of a set of SNP markers for population genetics of the red gorgonian (Paramuricea clavata), an emblematic species of the Mediterranean coralligenous. Conservation Genetics Resources, 2020, 12 (4), pp.521-523. 10.1007/s12686-020-011397. hal-03019888

\section{HAL Id: hal-03019888 \\ https: / hal.sorbonne-universite.fr/hal-03019888}

Submitted on 23 Nov 2020

HAL is a multi-disciplinary open access archive for the deposit and dissemination of scientific research documents, whether they are published or not. The documents may come from teaching and research institutions in France or abroad, or from public or private research centers.
L'archive ouverte pluridisciplinaire HAL, est destinée au dépôt et à la diffusion de documents scientifiques de niveau recherche, publiés ou non, émanant des établissements d'enseignement et de recherche français ou étrangers, des laboratoires publics ou privés. 
1 Development of a set of SNP markers for population genetics of the red gorgonian

2 (Paramuricea clavata), an emblematic species of the Mediterranean coralligenous

Padrón, $\mathrm{M}^{1}$., Milhes, M. ${ }^{2}$, Massot, M. ${ }^{3}$ \& Guichoux, E. ${ }^{3}$

$5{ }^{1}$ Laboratoire d'Ecogeochimie des Environnements Benthique, CNRS, Sorbonne Université, 6 UMR 8222, F-66650, Banyuls-sur-Mer, France

$7 \quad 2$ INRA, GeT-PlaGe, Genotoul, F-31326, Castanet-Tolosan, France

$8 \quad{ }^{2}$ Univ. Bordeaux, INRAE, BIOGECO, F-33610 Cestas, France

9

10 Correspondence email: padron@obs-banyuls.fr

Abstract

Transcriptome sequencing was used for the development of single nucleotide polymorphisms (SNP) for the red gorgonian (Paramuricea clavata). A total of 20,736 SNPs were identified, and 1,718 had a coverage of over 100 reads. Of the 480 SNPs tested, 347 SNPs were successfully genotyped at 95 samples from the NW Mediterranean using a MassARRAY System. This set of markers will be of great value for population genetics and phylogeography.

Keywords

Paramuricea clavata, Single nucleotide polymorphism, MassARRAY

\section{Introduction}

23 The gorgonian Paramuricea clavata is one of the most emblematic and conspicuous 24 coralligenous species in the Mediterranean Sea. A species of great interest for conservation 25 given its role in the persistence and stability of several associated species (Ballesteros 2006). 
26 However, given its slow dynamics, $P$. clavata is particularly vulnerable to disturbances. The red gorgonian has suffered the most drastic reductions in density and biomass over a broad spatial scale, as a consequence of sea temperature anomalies in the NW Mediterranean (Cupido et al. 2009; Santangelo et al. 2015). It is, therefore, essential to provide an understanding of the natural genetic variability of $P$. clavata populations, as well as their ability to withstand perturbations. The genetic structure of $P$. clavata populations has usually been studied with the use of a few microsatellite markers, sometimes resulting in conflicting results (Pilczynska et al. 2016; Padrón et al. 2018), and thus motivating the development of SNP markers for the species. Here, we describe a set of 118 SNP markers developed for population genetics of $P$. clavata, that were analyzed using iPLEX Gold technology on a MassARRAY System (Agena Bioscience, San Diego, USA).

\section{Material and Methods}

Transcriptome sequencing

Total RNA from ten individuals was used to sequence the transcriptome of $P$. clavata, using paired-end Illumina HiSeq 3000. RNAseq was performed at the GeT-PlaGe core facility, INRA Toulouse. RNA-seq libraries were prepared according to Illumina's protocols using the Illumina TruSeq Stranded mRNA sample prep kit to analyze mRNA. Briefly, mRNA was selected using poly-T beads. Then, RNA were fragmented to generate double stranded cDNA and adaptators were ligated to be sequenced. 11 cycles of PCR were applied to amplify libraries. Library quality was assessed using a Fragment Analyser and libraries were quantified by QPCR using the Kapa Library Quantification Kit. RNA-seq experiments have been performed on an Illumina HiSeq3000 using a paired-end read length of 2x150 pb with the 
Marker development and screening

The quality of the raw Illumina sequence reads was initially assessed using FastQC v0.10.1 (Andrews 2010). Based on the analysis report, Trimmomatic v0.32 was used to remove all the low quality reads with a Phred score below 20, as well as the Illumina adapters (Bolger et al. 2014). FastQC was performed again to verify the integrity of the remaining raw Illumina sequence reads. Given the lack of a reference genome for the species, high-quality reads were then used for the de novo transcriptome assembly, using Trinity with its default $k$-mer value of 25 (Grabherr et al. 2011). DiscoSnp++ v2.2.10 (Uricaru et al. 2015) was then used with default parameters to call SNPs using the assembled transcriptome as a reference.

A total of 1,718 SNPs with good coverage ( $>100$ reads) were identified from the transcriptome of $P$. clavata and submitted for assay design using the MassARRAY Assay Designer version 4.0.0.2 (Agena Biosciences). Genomic DNA was extracted from 95 colonies of the red gorgonian P. clavata sampled at different locations within the Ligurian Sea (Table 1). Twelve multiplexes of 40 SNPs, for a total of 480 SNPs, were designed for the genotyping which was performed on the 95 samples using the iPLEX Gold chemistry following Gabriel et al. (2009) on a MassARRAY System (Agena Biosciences). Allele calling was carried out with Typer Viewer v.4.0.24.71 (Agena Bioscience). We filtered out all monomorphic SNPs, loci with weak or ambiguous signal (i.e., displaying more than three clusters of genotypes or unclear cluster delimitation) and loci with too much missing data. The genetic parameters, including observed heterozygosity, expected heterozygosity, and the Hardy-Weinberg equilibrium (HWE), were calculated using GenoDive 2.0b23 (Meirmans and van Tienderen, 2004).

\section{Results}


76 A total of 347 SNPs were successfully amplified, of which 303 loci were polymorphic, resulting in a detectable rate of $87.32 \%$. The observed heterozygosity ranged from 0 to 1 , while the expected heterozygosity varied from 0.007 to 0.5 . 204 loci were significantly departed from HWE $(\mathrm{p}<0.01)$ after Bonferroni's correction (Supplementary Material). In summary, this is the first report of SNP markers in P. clavata developed using transcriptome and MassARRAY analysis. These SNPs would provide an invaluable resource for conservation genetics.

\section{Acknowledgements}

This work was funded by EC Interreg Marittimo program under project IMPACT - IMpatto Portuale su aree marine protette: Azioni Cooperative Transfrontaliere (PI, M. Magaldi; Ecology Task Leader, K. Guizien; CUP B12F17000370005). We thank the scuba divers, JC Roca and B Hesse, for their technical assistance for field sampling. RNA extractions were performed using instruments and facilities of the Marine Biodiversity and Biotechnology (Bio2Mar) platform of the OOB. SNP genotyping were performed at the Genome Transcriptome Facility of Bordeaux (grants from ANR-10-EQPX-16) with the help of Adline Delcamp and Christophe Boury.

\section{References}

Andrews S (2010) FastQC: a quality control tool for high throughput sequence data. Available online at: http://www.bioinformatics.babraham.ac.uk/projects/fastqc

Ballesteros E (2006) Mediterranean coralligenous assemblages: a synthesis of present knowledge. Oceanography and Marine Biology 44:1-74.

Bolger AM, Lohse M, Usadel B (2014) Trimmomatic: A flexible trimmer for Illumina Sequence Data. Bioinformatics 30(15): 2114-2120. 10.1093/bioinformatics/btu170 
100 Cupido R, Cocito S, Barsanti M, Sgorbini S, Peirano A, and Santangelo G (2009) Unexpected

101 long-term population dynamics in a canopy-forming gorgonian coral following mass mortality.

102 Marine Ecology Progress Series 394, 195-200.

103 Gabriel S, Ziaugra L, Tabbaa D (2009) SNP genotyping using Sequenom MassARRAY iPLEX

104 platform. Curr Protoc Hum Genet, Chapter 2: Unit 2.12.

105 Grabherr M, Haas B, Yassour M, Levin J, Thompson D, Amit I, Adiconis X, Fan L, 106 Raychowdhury R, Zeng Q, Chen Z, Mauceli E, Hacohen N, Gnirke A, Rhind N, di Palma F,

107 Birren BW, Nusbaum C, Lindblad-Toh K, Friedman N, Regev A (2011) Full-length 108 transcriptome assembly from RNA-seq data without a reference genome. Nat Biotechnol. 2011

109 May 15;29(7):644-52. doi: 10.1038/nbt.1883. PubMed PMID: 21572440.

110 Meirmans P, and van Tienderen P (2004) GENOTYPE and GENODIVE: two programs for

111 the analysis of genetic diversity of asexual organisms. Molecular Ecology Resources 4(4), $792-$ 112794.

113 Padrón M, Costantini F, Bramanti L, Guizien K, and Abbiati M (2018) Genetic connectivity 114 supports recovery of gorgonian populations affected by climate change. Aquatic Conservation 115 DOI: $10.1002 /$ aqc.2912.

116 Pilczynska J, Cocito S, Boavida J, Serrão E, and Queiroga H (2016) Genetic diversity and local

117 connectivity in the Mediterranean red gorgonian coral after mass mortality events. PLoS ONE 118 11(3), 1-16.

119 Santangelo G, Cupido R, Cocito S, Bramanti L, Priori C, Erra F, and Iannelli M (2015) Effects

120 of increased mortality on gorgonian corals (Cnidaria, Octocorallia): different demographic 121 features may lead affected populations to unexpected recovery and new equilibrium points. 122 Hydrobiologia 1-17 
123 Uricaru R, Rizk G, Lacroix V, Quillery E, Plantard O, Chikhi R, Lemaitre C, Peterlongo

124 P (2014) Reference-free detection of isolated SNPs. Nucleic Acids

125 Research. doi:10.1093/nar/gku1187

126 
Supplementary Material - Characteristics of 347 SNP markers in Paramuricea clavata

\begin{tabular}{|c|c|c|c|c|c|}
\hline SNP ID & Alleles & Primer $\mathbf{F}$ & Primer $\mathbf{R}$ & Ho & $\mathrm{He}$ \\
\hline SNP_10270 & $/ \mathrm{A}$ & CGCTGGCGGAAGTATTTTAC & STCCTTGGAAACAATCCCTG & 0.279 & 0.242 \\
\hline SNP_10524 & $\mathrm{G} / \mathrm{A}$ & GATGGACGAAATGTTGAGC & CGATGGAGAATTCGAACGG & 0.176 & 0.151 \\
\hline SNP_10540 & $\mathrm{C} / \mathrm{T}$ & GTCTTCGTCCTTCTCATGC & GCTCTCGAACAAGAAGAAGC & & \\
\hline SNP_10599 & $\mathrm{A} / \mathrm{G}$ & GTCTGAGGATGCTAGACTAC & TTGCCAGCGAGATCTCTATC & & 0.127 \\
\hline SNP_10670 & $\mathrm{T} / \mathrm{C}$ & AGACGGCTGTGTTTGTGTTG & GTGGCACAGAACAATGACG & .279 & 0.242 \\
\hline SNP_10819 & $\mathrm{C} / \mathrm{T}$ & TCAGCGATATTTCCCTGCAC & GACCAGGATTTACACTGTTG & & \\
\hline SNP_10870 & $\mathrm{G} / \mathrm{C}$ & ACATTAGTCCACCACTCAAC & TTGACATGCCATACTTCGCC & & \\
\hline SNP_11020 & $\mathrm{C} / \mathrm{T}$ & GCAGTTTTCGGCACAACATC & TTTTCGACATGACTGTTGGC & & \\
\hline SNP_11054 & $\mathrm{G} / \mathrm{C}$ & ATTTCTGTTTCGGCGAGGTC & TATCGCTTCAGGGTCTACAG & & \\
\hline SNP_1123 & $\mathrm{C} / \mathrm{T}$ & TGGGAAATTTCTTACGGCTC & CTTTTCAGTCCCAGAAGACG & & \\
\hline SNP_11288 & $\mathrm{C} / \mathrm{T}$ & ATGCACTTGCCTGAATGCTG & ACCACCACCAAAGAGGAAAC & & \\
\hline SNP_11320 & $\mathrm{T} / \mathrm{C}$ & GTCTCGTTATCGTATCGACC & CCCTGTGGAAGAATATGACG & & \\
\hline SNP_11339 & $\mathrm{C} / \mathrm{T}$ & GGGACCAGAACAATTTAGAG & GTGTCTTGGTCGTAATAAGG & 0.477 & 0.420 \\
\hline & & & & & \\
\hline SNP_11394 & $\mathrm{A} / \mathrm{G}$ & TTCTC & ACATCA & 0.050 & 0.069 \\
\hline SNP_1146 & $\mathrm{G} / \mathrm{A}$ & & 171 & & \\
\hline SNP_11478 & $\mathrm{G} / \mathrm{A}$ & TGATCCCGAAACCAAGAAAC & CATATATCTAGCAGTACCCG & 89 & 0.422 \\
\hline SNP_11506 & $\mathrm{G} / \mathrm{C}$ & & CTGACACACAGATTAAAGAGG & & 0.292 \\
\hline & $\mathrm{A} / \mathrm{G}$ & ACG & $\mathrm{AC}$ & & \\
\hline SNP_11582 & $\mathrm{C} / \mathrm{T}$ & GATC & & & 0.000 \\
\hline & $\mathrm{G} / \mathrm{A}$ & СТTТСТСТ & TCCGT & & \\
\hline SNP_11619 & $\mathrm{G} / \mathrm{C}$ & AATGTCATGCTATCCAGCCG & GAATGGCGA & 0.300 & 0.300 \\
\hline & & & & & \\
\hline SNP_11770 & $\mathrm{A} / \mathrm{G}$ & ACACATCAGCT & AAGATACT & & \\
\hline SNP_11882 & $\mathrm{G} / \mathrm{A}$ & ATCGAC & GCCAC & 482 & 0.426 \\
\hline SNP_12083 & $\mathrm{T} / \mathrm{C}$ & GCTCGAAGATCAGCAGTT & GGTTTGATGGCA & & 0.017 \\
\hline SNP_12101 & $\mathrm{G} / \mathrm{A}$ & TGAAC & TTGCC & 602 & 0.480 \\
\hline SNP_12120 & $\mathrm{G} / \mathrm{A}$ & & CTTTGC & & 0.384 \\
\hline SNP_12259 & $\mathrm{G} / \mathrm{A}$ & AAGC & GCTC & & 0.032 \\
\hline & $\mathrm{A} / \mathrm{G}$ & ACCTTTGCGGCCTTCTTGTC & TTTGCCAGA & & \\
\hline SNP_12309 & $\mathrm{C} / \mathrm{T}$ & AGCGCCAAGTTATTTAAAG & CACGCTGATGAAAAATC & 0.2 & 0.235 \\
\hline & $\mathrm{C} / \mathrm{T}$ & & CGTATTGCATTGAAGCGTCC & & 0.356 \\
\hline SNP_12339 & $\mathrm{A} / \mathrm{T}$ & GACTCCACAAAAGTTCAAGAC & ATCACTCCCTCTAGACGTTC & & 0.313 \\
\hline SNP_1235 & $\mathrm{C} / \mathrm{T}$ & CTACCTCTTCGGCCATTTTC & TGTTTGAGTGGAGACTGTTC & 0.346 & 0.377 \\
\hline SNP_12396 & $\mathrm{C} / \mathrm{T}$ & CAAACTTGCGGAAACCGAAG & GCGTTGTTTAGCTTTCTCGG & 0.496 & 0.432 \\
\hline SNP_12408 & $\mathrm{C} / \mathrm{T}$ & GGACCAAGCATTGTTTCAGG & AATTCCTTTGCCAGTCGCTC & 0.171 & 0.184 \\
\hline SNP_12410 & $\mathrm{G} / \mathrm{A}$ & ATACACGCGAGTTGATCCAC & GACATGTGGAGTTTTGGATG & 0.344 & 0.331 \\
\hline SNP_12451 & $\mathrm{G} / \mathrm{C}$ & ATGCTTGTGGCGTAGTTTCG & GAGAGTGTTGGCTGAAGATG & 0.074 & 0.068 \\
\hline SNP_1253 & $\mathrm{C} / \mathrm{T}$ & AAAGAGCGAGAGGAAATGGC & TGATCCAAAGTTCTCCCGAC & & 0.352 \\
\hline SNP_12548 & $\mathrm{A} / \mathrm{G}$ & GTCAAATGATCACACTATCC & GTGTGCCAAAGGTTTTCCTG & 0.000 & 0.000 \\
\hline SNP_1256 & $\mathrm{C} / \mathrm{T}$ & ACATCCCTATTACCTCCGAC & TTGGTACTGACGTCGTTTGC & 0.415 & 0.332 \\
\hline SNP_12634 & $\mathrm{T} / \mathrm{C}$ & ACCATGTAGACACCCCAAAG & CTGAACACAATTTGGCTCAC & 0.065 & 0.117 \\
\hline SNP 12675 & $\mathrm{G} / \mathrm{A}$ & TGGAAACTCAAGCGGGAAAC & GTATCGTCTAGCGATCACAC & 0.364 & 0.345 \\
\hline
\end{tabular}




\begin{tabular}{|c|c|c|c|c|c|}
\hline SNP ID & Alleles & Primer F & Primer R & Ho & He \\
\hline SNP_12676 & $\mathrm{A} / \mathrm{G}$ & CTTCTGTCAACTTGAACGCC & TTCAGAGTGCTTGCCTGAAG & 0.046 & 0.071 \\
\hline SNP_12753 & $\mathrm{T} / \mathrm{C}$ & TTAACTGCGCAGAACGAGG & AGGCAGCAATTGTCATTCG & 146 & 0.121 \\
\hline SNP_1285 & $\mathrm{C} / \mathrm{A}$ & TCGTTGTGACTGTGAGCTAC & GCTGATCTCAATATGAACCC & 0.237 & 0.267 \\
\hline SNP_12854 & $\mathrm{A} / \mathrm{T}$ & CATGACGGAGCCTATTGATG & TCTGGCAACTCCAAACCTTC & 0.318 & 0.303 \\
\hline SNP_12925 & $\mathrm{A} / \mathrm{G}$ & GTCTGTTCTCTTCTTCCATC & AAACAACGTGCTACCCAGAG & 0.099 & 0.113 \\
\hline SNP_12958 & $\mathrm{G} / \mathrm{A}$ & ACCTAACTCGCTTTTCGTGG & TGACGTCAAGGAATTCTCGG & 0.393 & 0.351 \\
\hline SNP_13079 & $\mathrm{C} / \mathrm{T}$ & ACCTTGTTGCAAAGTCTGCC & AGGAATATGCAGCGAGGATG & 0.250 & 0.254 \\
\hline SNP_13227 & $\mathrm{G} / \mathrm{A}$ & CATCAAGCAGAACTTCCAGC & CCGAGCTGAACATACAGTTG & 0.169 & 0.153 \\
\hline SNP_13306 & $\mathrm{C} / \mathrm{T}$ & TCCTGAGCCGTGAAACATTC & AAGGTGAGTACACACTTCCC & 0.408 & 0.405 \\
\hline SNP_1332 & $\mathrm{A} / \mathrm{T}$ & GTTTATCGACATGGCCTTTG & CGTCCAAACATTTAGCC & 0.381 & 0.431 \\
\hline SNP_13353 & $\mathrm{C} / \mathrm{T}$ & ATATCAACGCATCCAGGTCG & CACTCTCCTGTGAATCAGC & 0.323 & 0.343 \\
\hline SNP_13401 & $\mathrm{G} / \mathrm{A}$ & TTCCTTCGCAAGGCTTGTTG & AAGGCTTCGAATCGCACA & 0.000 & 0.408 \\
\hline SNP_13505 & $\mathrm{C} / \mathrm{T}$ & ACAAACGGGTCCTGTGTTTC & GGGAGAGTATGTAGAAGTTG & 0.400 & 0.357 \\
\hline SNP_13634 & $\mathrm{G} / \mathrm{A}$ & AAGACATGGTCGAAGCGATG & CATTCTTGTAGGCAACCGAC & 0.019 & 0.038 \\
\hline SNP_13693 & $\mathrm{C} / \mathrm{T}$ & СCTTTTGACTTTACATCTTCC & GAGCGAACGAGTTGAAAAGG & & 0.100 \\
\hline SNP_13760 & $\mathrm{C} / \mathrm{T}$ & CCAGTCAGA & GTCC & 0.101 & 0.195 \\
\hline SNP_13806 & $\mathrm{C} / \mathrm{A}$ & CTTTCACTGTTTG & GGCA & 0.000 & 0.234 \\
\hline SNP_13855 & $\mathrm{A} / \mathrm{G}$ & AATTCCTGTTTCATCTCGCC & & & \\
\hline SNP_1388 & $\mathrm{T} / \mathrm{C}$ & GCCACGTTGAGTAGATTCAG & CTGCAGACTGTCTTTGTTCG & 0.084 & 0.067 \\
\hline SNP_13901 & $\mathrm{C} / \mathrm{A}$ & CCAGTACAACGCCCTTAATC & TTTCTTC $\mathrm{GCC}$ & 211 & 0.229 \\
\hline SNP_13908 & $\mathrm{C} / \mathrm{A}$ & ATCGCCAA & GCGA & 0.000 & 0.105 \\
\hline SNP_13932 & $\mathrm{G} / \mathrm{T}$ & GGCTGTTT & TTACA & 0.284 & 0.330 \\
\hline SNP_13981 & $\mathrm{G} / \mathrm{A}$ & GCTATGGATGGTACAGAAGG & AACTGGGATGGTGATTGGTC & 0.304 & 0.273 \\
\hline SNP_13993 & $\mathrm{C} / \mathrm{T}$ & CATGTACTCGTGGCTTTCTC & CGTTGACGAACTTCGAAAGG & 0.195 & 0.194 \\
\hline SNP_14046 & $\mathrm{C} / \mathrm{T}$ & GACGTT & & 0.6 & 0.407 \\
\hline SNP_14275 & $\mathrm{T} / \mathrm{C}$ & TGTACT & $\mathrm{G}$ & 0.031 & 0.027 \\
\hline SNP_14312 & $\mathrm{C} / \mathrm{A}$ & TAAGCTTGTCCAGCAGTAGG & GGCAGT & 0.323 & 0.309 \\
\hline SNP_14315 & $\mathrm{G} / \mathrm{T}$ & ATTTCGACGTCCAGAGACAG & ГCCGGTCTTATTGATG & 0.000 & 0.000 \\
\hline & & & & & 0.394 \\
\hline SNP_14492 & $\mathrm{T} / \mathrm{C}$ & GTGCTAACATCTTCCCAAGG & GATCCGCATGGATAAG & 0.480 & 0.415 \\
\hline SNP_14496 & $\mathrm{G} / \mathrm{A}$ & TAAACTCTGCCTACGGACTG & TTCCGCCCACAGATATCAAC & 0.173 & 0.204 \\
\hline SNP_14523 & $\mathrm{T} / \mathrm{C}$ & GGCAAAGTTTGACCGAAATG & ATATCTTCGGGACGACGAAC & 0.345 & 0.363 \\
\hline SNP_14565 & $\mathrm{G} / \mathrm{A}$ & TCACATGGTGGATGAGTTGG & AGTGCCTCCCCATTCTTTTC & 0.270 & 0.279 \\
\hline SNP_14570 & $\mathrm{T} / \mathrm{A}$ & CAATTGTCAAAAGGAGGGTC & TCACCTCGTGAATCCTCCTC & 0.113 & 0.098 \\
\hline SNP_14598 & $\mathrm{A} / \mathrm{G}$ & CAATGCGGAGGAGTGATGAA & AACCTCCATCTGTCCATCAC & 0.393 & 0.378 \\
\hline SNP_14698 & $\mathrm{C} / \mathrm{T}$ & GAGCAAGTTTGCTTTTAGATG & AGAACCAAAACAGGCAAAGC & 0.423 & 0.434 \\
\hline SNP_14739 & $\mathrm{A} / \mathrm{G}$ & AAATCGGAACCGAATTGGGC & GCTATGAAACAGGTTGCAGG & 0.036 & 0.031 \\
\hline & $\mathrm{G} / \mathrm{C}$ & GAAAGTACTGACTGCATGCC & & 0.307 & 0.412 \\
\hline SNP_14809 & $\mathrm{T} / \mathrm{A}$ & AGGCAACATCGAAGGTCTAC & TTCACAGCCGATTCAACGAG & 0.491 & 0.435 \\
\hline SNP_14896 & $\mathrm{T} / \mathrm{C}$ & TGGGCATGTAGTAATCCTTG & AACTGAACCTTTGCCAGCTC & 0.221 & 0.191 \\
\hline SNP_14929 & $\mathrm{G} / \mathrm{T}$ & GAGTCAAACCATTGTTGACC & TGGTCAACCGCAACAACAG & 0.343 & 0.414 \\
\hline SNP_15089 & $\mathrm{T} / \mathrm{C}$ & GCTGAACAAAATTCCCTCGG & GCTATAAАССТTTCCTTCAC & 0.208 & 0.176 \\
\hline SNP_15124 & $\mathrm{C} / \mathrm{G}$ & GGCCCTTTTCCAATTCGTTC & TCTAGCACTTAGCGACTGCC & 0.161 & 0.164 \\
\hline SNP_15221 & $\mathrm{T} / \mathrm{C}$ & CCGAGAAAAATGATGTCGTTG & CGACAGATATTGCAGTAAAG & 0.431 & 0.394 \\
\hline SNP_15231 & $\mathrm{G} / \mathrm{A}$ & TTCTGCAGAGAAAGCCTACC & TGGCTGGTTCAAAACACGAG & 0.069 & 0.064 \\
\hline
\end{tabular}




\begin{tabular}{|c|c|c|c|c|c|}
\hline SNP ID & Alleles & Primer F & Primer R & Но & $\mathrm{He}$ \\
\hline SNP_15393 & $\mathrm{G} / \mathrm{T}$ & CCAAAAACGCAGAGATATCC & TGTGCTTCTTGGAAAATCTC & 0.457 & 0.402 \\
\hline SNP_15479 & $\mathrm{G} / \mathrm{A}$ & CTAAAGGGCAGTAAACCATC & GGTCGGTCATGTAGAAATC & & \\
\hline SNP_15519 & $\mathrm{T} / \mathrm{C}$ & TTGCAGATCTTGTTGTTGGC & GTACTTTGCCAAGCGTTCTG & 0.364 & 0.346 \\
\hline SNP_15610 & $\mathrm{A} / \mathrm{G}$ & ACGACTGTGTGGAAGAACTG & CATCGCTGTACCAAATGGTG & 0.272 & 0.262 \\
\hline SNP_15651 & $\mathrm{T} / \mathrm{C}$ & TGGTAAATCAGTCGTCTTGG & GCTTCTTCAACAACTTTGAG & 0.423 & 0.397 \\
\hline SNP_15809 & $\mathrm{T} / \mathrm{A}$ & ATCACTCTGTTCTTCCGTTG & AGGCCAAGTTGACGAAGATG & 0.312 & 0.324 \\
\hline SNP_15907 & $\mathrm{G} / \mathrm{A}$ & AACTGCTCTTCTGAACATGG & TTGAACCTGTCATGATGAAC & 0.203 & 0.184 \\
\hline SNP_15911 & $\mathrm{C} / \mathrm{T}$ & GGCAAAATTTCATTGTCGGC & TGCAAATGCTCAGTTGAGGG & & \\
\hline SNP_16021 & $\mathrm{A} / \mathrm{G}$ & GGCCACCTCTAAGAACAAAC & GGTTGGATCGTTTCTAGAAC & 0.203 & 0.227 \\
\hline SNP_16079 & $\mathrm{G} / \mathrm{A}$ & AAGTGCCCGTTCTTCGATAG & CGACCAGTTGTTTTACCCAC & 0.087 & 0.078 \\
\hline SNP_16208 & $\mathrm{G} / \mathrm{A}$ & GTACCCTTCGACGATTTCTC & TTGGCAAGGAGAAGCTTGAC & 0.000 & 0.000 \\
\hline SNP_16249 & $\mathrm{G} / \mathrm{A}$ & TGTGTCCGTCGTTTTGTTCC & TGGATAGATGCAGGCAGTAG & 0.361 & 0.346 \\
\hline SNP_16318 & $\mathrm{C} / \mathrm{T}$ & TGGACCACATGATTCACA & ATGGTTAGAG & 0.296 & 0.387 \\
\hline SNP_16335 & $\mathrm{T} / \mathrm{C}$ & TTGGGAGATTGCCGGTAC & ССТАСТТС & 0.100 & 0.083 \\
\hline SNP_16476 & $\mathrm{C} / \mathrm{T}$ & AGCCACCTTAACT & GCTGGCCCTTTTTGTATTGG & 0.333 & 0.390 \\
\hline SNP_16583 & $\mathrm{G} / \mathrm{T}$ & CACTGCTTGCCTTGAACATC & ATCACTTGCAGATCTGCAGG & 0.070 & 0.059 \\
\hline SNP_16598 & $\mathrm{G} / \mathrm{A}$ & CGTCATGCATGTGAAACTTG & TGGTGAAGACGATGATGGAC & 0.499 & 0.451 \\
\hline SNP_16611 & $\mathrm{C} / \mathrm{G}$ & TGGTGCCTGTTACAACTGTG & TGAGACTCTCTCTTTAGT & 0.362 & 0.326 \\
\hline SNP_16676 & $\mathrm{T} / \mathrm{C}$ & AGCATGGTCTATAGGTGGAG & TAAAACCAGCTGCTCCCA & 0.406 & 0.382 \\
\hline SNP_16687 & $\mathrm{G} / \mathrm{A}$ & ССТТТССТТСТТССТСААСС & CTCACAGGCAAATAGGCT & 0.306 & \\
\hline SNP_16955 & $\mathrm{G} / \mathrm{A}$ & CAAAATTCGACCACTCTGGC & TGCTCGTTCCCTATCGTTAC & 0.358 & 0.352 \\
\hline SNP_17055 & $\mathrm{C} / \mathrm{T}$ & & & & 0.410 \\
\hline SNP_17060 & $\mathrm{G} / \mathrm{T}$ & TCAGCTCCAATGGT & TTAGCCAT & 0.236 & 0.187 \\
\hline SNP_17162 & $\mathrm{G} / \mathrm{A}$ & CTGGGAAGTA & TTGGAAGC & 0.442 & 0.378 \\
\hline SNP_17172 & $\mathrm{G} / \mathrm{A}$ & GCATTTGGTCACCTTAGCAG & TACCGAGGCGAAAATCTGTC & 0.358 & 0.334 \\
\hline SNP_17179 & $\mathrm{G} / \mathrm{A}$ & ATTTGATTTACTGGACGAGG & СТСТССТTGCAAATTAAAAAG & 0.251 & 0.202 \\
\hline SNP_17184 & $\mathrm{A} / \mathrm{G}$ & CAAAGTACACGGCAGGATTG & TGATCGTGAAATGTCTGGAG & 0.207 & 0.294 \\
\hline SNP_17190 & $\mathrm{T} / \mathrm{C}$ & GGCACTGCATCAAAATCTTC & CGGACATTG & 0.183 & 0.154 \\
\hline & $\mathrm{A} / \mathrm{G}$ & GTGATGTGATTATGCTTTCGG & AATCGTGAACACT & & \\
\hline SNP_17351 & $\mathrm{C} / \mathrm{T}$ & CCATATCGGCATCATACTTC & GAACGCGAAAAGAGGAAGAG & 0.288 & 0.309 \\
\hline SNP_17412 & $\mathrm{G} / \mathrm{C}$ & TAGCTTTAGTCTGTGCAGCG & CTTCAAAGGTATTCGACCCG & & 0.300 \\
\hline SNP_17451 & $\mathrm{T} / \mathrm{A}$ & TGATCTTGCGACAGTCACTC & ATCAACGCCTTCCAAGTTCC & 19 & 0.242 \\
\hline SNP_1746 & $\mathrm{T} / \mathrm{A}$ & TTTTCTGGATCGATGTCGTC & TTGAAGGCGTTATTGCCTCC & 0.223 & 0.420 \\
\hline SNP_17519 & $\mathrm{T} / \mathrm{A}$ & ATGCAGCACATTGTTCCTCC & TCAGATGACGTCACATCCTC & 0.347 & 0.308 \\
\hline SNP_17549 & $\mathrm{G} / \mathrm{A}$ & TCGGAGACACAGCTTACAAC & GACTTAGCTTCTGTTTTCGG & 0.279 & 0.362 \\
\hline SNP_17570 & $\mathrm{C} / \mathrm{T}$ & CGCAACACCATAACCTTTCC & CGGCAGTGGAAAACAGAGTT & 0.286 & 0.212 \\
\hline SNP_17745 & $\mathrm{A} / \mathrm{G}$ & TGTTCCGTCGCAAAGCTTTC & TTCATGTTTGATTCAGCCTC & 0.138 & 0.119 \\
\hline SNP_17771 & $\mathrm{T} / \mathrm{C}$ & CATCCTTCGTCGTCTTTTCC & TAGGTCACTATCACACGCTG & & 0.244 \\
\hline SNP_17782 & $\mathrm{C} / \mathrm{T}$ & TCGCAAGCGTTGGAGTATTG & TTGTCTCCCTGCTTGGAAAG & 0.325 & 0.284 \\
\hline & $\mathrm{A} / \mathrm{G}$ & TGAAGGATCGATCCCAGTTG & TGAAGGCTGTGGATGGACTC & 0.208 & 0.340 \\
\hline SNP_17854 & $\mathrm{T} / \mathrm{A}$ & GTGGATTGATCCTGATGAAG & GGTTCTCCAATTCGCCATAT & 0.521 & 0.455 \\
\hline SNP_17907 & $\mathrm{C} / \mathrm{T}$ & СCTCTCATGCGCAGAACTTC & TGGCTATCCCATGCAACAAC & 0.401 & 0.409 \\
\hline SNP_17944 & $\mathrm{G} / \mathrm{A}$ & GATCTCCCAACTTTCTGACG & ATATTTGGAGGCTAACGCGG & 0.270 & 0.244 \\
\hline SNP_17967 & $\mathrm{G} / \mathrm{A}$ & CACCCTGGATTCTCTTCATC & CGAGTATGTGAGGAAATTGC & 0.229 & 0.205 \\
\hline SNP 1804 & $\mathrm{~A} / \mathrm{T}$ & ACCCTGTTGCTTTGGGTATC & GAAACGACCTTTGTCGTTGG & 0.052 & 0.064 \\
\hline
\end{tabular}




\begin{tabular}{|c|c|c|c|c|c|}
\hline SNP ID & Alleles & Primer F & Primer R & Ho & He \\
\hline SNP_18058 & $\mathrm{C} / \mathrm{A}$ & TCTGAACCCTCCTCTGAATC & GGAACAAGAAATTGAAGACG & 0.071 & 0.065 \\
\hline SNP_18067 & $\mathrm{T} / \mathrm{C}$ & AAGCATCCAGAATACTGCG & CAATACGTGATACCCTGAG & 700 & 0.415 \\
\hline SNP_18076 & $\mathrm{A} / \mathrm{G}$ & GTAAGAAAGGTTACCATGGC & TTGACAATTCGCCTGAGCTG & 0.188 & \\
\hline SNP_18088 & $\mathrm{G} / \mathrm{A}$ & GATCTTCTTTCATGTAGAG & GGTGGATCTTTCACTGTTGC & 0.411 & 0.412 \\
\hline SNP_18374 & $\mathrm{T} / \mathrm{C}$ & CGGAATGCTGCTTGAATCTG & GAAATTTGCCGCCCATACTG & 0.051 & \\
\hline SNP_18402 & $\mathrm{C} / \mathrm{A}$ & AGCATGCCATCTCAACAACC & GTTGGAGTACTTCTGCTTGG & 0.319 & 0.364 \\
\hline SNP_18404 & A/ G & TGCCACACGAGTTGTGATG & GCGACACGTTTTTACGATCC & 0.093 & 0.075 \\
\hline SNP_18524 & $\mathrm{T} / \mathrm{C}$ & TGGCTAAAGCTGCAAAAGCG & TTGTCGCCACATGGTATGAG & 0.152 & 0.154 \\
\hline SNP_1860 & $\mathrm{G} / \mathrm{A}$ & ATCGGAATTCCTTCTCAATC & AAGGCGACGATAAGTCTCGG & 0.000 & 0.083 \\
\hline SNP_18639 & $\mathrm{G} / \mathrm{A}$ & TAGACGAATGAGGTTCTGCG & ATAGAGGAGGACAAAGGTGG & 0.411 & 0.414 \\
\hline SNP_18674 & $\mathrm{T} / \mathrm{A}$ & TGATCTTCCTTTCCTTTTG & ICTGAAGAACTCGATGAGGG & & 0.280 \\
\hline SNP_18715 & $\mathrm{A} / \mathrm{G}$ & GAAACAGCGCCTTGAAGAAC & AGTGGTTGTCATGGTGACAG & 0.608 & 0.481 \\
\hline SNP_18797 & $\mathrm{T} / \mathrm{C}$ & AACCAACCAGCGAACTTCAC & TCTTCGCTGGTCCTTCAATC & 0.031 & 0.036 \\
\hline SNP_18805 & $\mathrm{T} / \mathrm{C}$ & AGGTTCAATATGGTGGACGG & GTCAGTAAACCAGACATGGG & 0.404 & 0.445 \\
\hline SNP_18834 & $\mathrm{A} / \mathrm{T}$ & GCAAGAAAAAGTCGCCCAAG & CCTCTTTTCGTTGTCGAT & 0.938 & 0.490 \\
\hline SNP_18847 & $\mathrm{G} / \mathrm{A}$ & GACAAAATGCTCGACATGCC & CAATTTGTGGAGC & 0.445 & 0.397 \\
\hline SNP_18991 & $\mathrm{C} / \mathrm{T}$ & GATACTTTCAGATCCCCAGG & AGAATCCACCCTGTGAGAAC & 0.494 & 0.405 \\
\hline & & & TCTCTTGGTTTTGTTTCCCG & & \\
\hline SNP_19078 & $\mathrm{G} / \mathrm{A}$ & GGCTCAACTCCAAGATCTTC & CTCGTCTAACACGGGATATG & 0.505 & 0.380 \\
\hline SNP_19104 & $\mathrm{C} / \mathrm{T}$ & AACGTTTTAGTCCTCGATAG & CTTAGTGATCACATTTGAC & 0.087 & 0.112 \\
\hline SNP_19116 & $\mathrm{T} / \mathrm{C}$ & ATTTGAGCGTGT & TATC & 0.319 & 0.332 \\
\hline SNP_19125 & $\mathrm{G} / \mathrm{A}$ & AAGATGATACAC & & 0.117 & 0.104 \\
\hline SNP_19126 & $\mathrm{A} / \mathrm{G}$ & AАААТСТССССТССТССТTC & GCTACTGACGTCAGCATTTG & 0.237 & 0.223 \\
\hline SNP_19147 & $\mathrm{C} / \mathrm{T}$ & GACATCATAGCCGTGATTGG & ATG & 0.367 & 0.402 \\
\hline SNP_19267 & $\mathrm{C} / \mathrm{T}$ & СТСССТС & GGGTGATATCT & & 0.354 \\
\hline SNP_19277 & $\mathrm{C} / \mathrm{T}$ & ATGATGTGTTGAGATGCGCC & TCCGAGTTTATGCACACATC & 0.120 & 0.118 \\
\hline & $\mathrm{A} / \mathrm{G}$ & CGGAAGAGACGCTGTTATTG & CCAGCCAAAGTACAAGAGAG & & \\
\hline SNP_1954 & $\mathrm{T} / \mathrm{C}$ & TACAGAGTCGTATTCG & ATCCTCAAATGCCTT & 0.486 & 0.374 \\
\hline SNP_1960 & $\mathrm{C} / \mathrm{T}$ & GAGATCTCTGATCAGACGAC & CGTCTCAGTATGCCATACAG & 0.219 & 0.208 \\
\hline SNP_19609 & $\mathrm{C} / \mathrm{T}$ & AGAAGCTTCTCAAGCTGATG & & 0.197 & 0.170 \\
\hline SNP_19646 & $\mathrm{C} / \mathrm{T}$ & GAAGAGATCGCCATTGACAG & AGCAGAAGCGATGCTAAATG & 0.356 & 0.367 \\
\hline SNP_19749 & $\mathrm{G} / \mathrm{A}$ & CCAGATTCAGCTTCTCCAAC & CAACGGAGAGTAAAGAGAGC & 0.000 & 0.000 \\
\hline SNP_19926 & $\mathrm{A} / \mathrm{G}$ & ACATGCAACACTGTTTCAGG & TGCAGGAGATTTTGGATGAC & 0.287 & 0.327 \\
\hline SNP_19945 & $\mathrm{C} / \mathrm{T}$ & CACACTCTTGTGTAAATTGG & AGTTCCCTCTTGCTAAAGAC & 0.232 & 0.419 \\
\hline SNP_19956 & $\mathrm{A} / \mathrm{G}$ & CCGTGAGTTTTTGCGTTTCG & CTTCACATCAGCTCGAGTTC & 0.178 & 0.335 \\
\hline & $\mathrm{G} / \mathrm{A}$ & GGCTTGAAACCAATACCTCG & & & 0.345 \\
\hline SNP_20077 & $\mathrm{C} / \mathrm{T}$ & AACAATCCACCATCGATACC & GTTTAATGAAAAGCCACGCC & 0.608 & 0.481 \\
\hline SNP_20191 & $\mathrm{C} / \mathrm{T}$ & CTGGCATTCTGAGTTAATGG & TCAGACCTCTTCACAGAGAC & 0.411 & 0.327 \\
\hline SNP_20242 & $\mathrm{G} / \mathrm{T}$ & GTCAAAGTAGCAACTGCGTG & TACACTGCCATTTCAGTGGG & 0.351 & 0.373 \\
\hline SNP_20340 & $\mathrm{A} / \mathrm{G}$ & TTCCCCCCAGCGTTAACAAC & TCTTCGACGCTAACTATGAC & 0.141 & 0.113 \\
\hline SNP_2041 & $\mathrm{G} / \mathrm{C}$ & TAATGCGCCTGGTTTTCCTC & TGTTGACGTCACTGGTTCAC & 0.055 & 0.085 \\
\hline SNP_20462 & $\mathrm{A} / \mathrm{G}$ & GTTGTACTCCAATGGTTGTG & GGAACATATCGGTTCATGTTG & 0.424 & 0.383 \\
\hline SNP_20742 & $\mathrm{C} / \mathrm{T}$ & TCCCCCATCTGATATCTCTC & TGACAGTGACGTAGTTCTGG & 0.117 & 0.208 \\
\hline SNP_20778 & $\mathrm{C} / \mathrm{T}$ & ATGCATCCCAGACATGTTCC & AAGTCGTAATGCGAGTTGGG & 0.485 & 0.392 \\
\hline SNP_20807 & $\mathrm{C} / \mathrm{T}$ & TCTTGTAGAACCACGTCGAG & ATACAACAACGCCTGGGAAC & 0.213 & 0.191 \\
\hline
\end{tabular}




\begin{tabular}{|c|c|c|c|c|c|}
\hline SNP ID & Alleles & Primer F & Primer R & Но & $\mathrm{He}$ \\
\hline SNP_20813 & $\mathrm{i}$ & AGCTGTTGATATCCTAGCTG & AGATAACACCTCGCTCTCTC & 0.000 & 0.000 \\
\hline SNP_20823 & & ATGCCACGTCGTACGATTTC & CTGTTCCATTAACAGGAGG & & \\
\hline SNP_20856 & $\mathrm{T} / \mathrm{C}$ & CCTCATTGTTTTATCCGCTG & CGTTTATGTCTTCCCTCACC & .321 & 0.260 \\
\hline SNP_20911 & $\mathrm{A} / \mathrm{G}$ & TTCGTCGGAGAGCATCTTTG & CTGGCTGTGGTTTTTATGG & .413 & 0.439 \\
\hline SNP_2093 & $\mathrm{C} / \mathrm{G}$ & GTGAAGACACTGACTGACTC & TGGTGTTTCCCTCAGTGATG & 0.102 & 0.111 \\
\hline SNP_21016 & $\mathrm{G} / \mathrm{A}$ & TCATCATGGCCATCTTCACC & ATGTGGTCCATGCGTGTTTC & 0.423 & 0.397 \\
\hline SNP_21049 & $\mathrm{G} / \mathrm{A}$ & TCAGCCAAGAAATCAACGGG & GCCAACATTATCTCAAGGTC & 0.436 & 0.437 \\
\hline SNP_21091 & $\mathrm{G} / \mathrm{A}$ & ATCCAGTTGCTTCTTTGTCG & TCTACCATCTTCTGACGGAG & 0.246 & 0.255 \\
\hline SNP_21257 & $\mathrm{G} / \mathrm{A}$ & СTCTTCGACAAATACGTACC & GTCTGGTATAGGTGTGATCT & 0.450 & 0.368 \\
\hline SNP_21270 & $\mathrm{G} / \mathrm{A}$ & ATCAATACCAGCAACCAAGG & GGCGTAAGGCAGTAATTGTG & 0.392 & 0.370 \\
\hline SNP_21417 & $\mathrm{G} / \mathrm{A}$ & TAGTACCATATGCTCTTCTG & ATGTACGATGCCGATGAGAC & 0.164 & 0.357 \\
\hline SNP_21481 & $\mathrm{C} / \mathrm{T}$ & AATACACAAGATCGCTGAGG & AGTGGCATTTTCTCCACAGG & 0.282 & 0.274 \\
\hline SNP_21512 & $\mathrm{C} / \mathrm{A}$ & TCAGTACTGCGGAAT & ACTGCTAT & 0.500 & 0.454 \\
\hline SNP_21554 & $\mathrm{G} / \mathrm{A}$ & TCAACCTGGG & TGTCCATC & 0.621 & 0.480 \\
\hline SNP_21565 & $\mathrm{G} / \mathrm{A}$ & TGTTACAATCCCACGACGAG & TATCTTCT & & 0.176 \\
\hline SNP_2181 & $\mathrm{C} / \mathrm{G}$ & AGACCATCCGTCGCCATTC & CGTCATACTCTGCAATCCTC & 0.144 & 0.144 \\
\hline SNP_21828 & $\mathrm{C} / \mathrm{T}$ & CCTTGTCCTGTACTGCAATC & TTAGATGTCGACTGTTCAGG & 0.320 & 0.320 \\
\hline SNP_21872 & $\mathrm{A} / \mathrm{G}$ & AAGGCCAAACGATGATGAGG & TTGTGACGACTCAGCTAC & 0.431 & 0.431 \\
\hline SNP_21884 & $\mathrm{C} / \mathrm{G}$ & AATTCCTCCCGCATGAGTTC & TAGTCAAGGAAGCCAAGAGG & 95 & 0.083 \\
\hline SNP_21937 & $\mathrm{C} / \mathrm{T}$ & TTCTGAAAGACCAGGTGCTC & TGTGCCAAGCGTTGCTTTTC & & 0.177 \\
\hline SNP_21973 & $\mathrm{C} / \mathrm{T}$ & GAAAGGCGTTCCTTAGCTTG & TGTGAGACGTTCATCTGCTC & 0.000 & 0.000 \\
\hline SNP_21974 & & & & & \\
\hline SNP_21976 & $\mathrm{C} / \mathrm{T}$ & TCTC & AATGAT & 50 & 0.140 \\
\hline SNP_22047 & $\mathrm{C} / \mathrm{T}$ & TATCCT & TCGATG & 0.479 & 0.448 \\
\hline SNP_22128 & $\mathrm{T} / \mathrm{C}$ & TAGAGATATCTTTGTAGCG & GTGATGAAAGAACACGAAAG & 0.366 & 0.347 \\
\hline SNP_22185 & $\mathrm{C} / \mathrm{A}$ & CGCTTGAGTTTTTGTAACGG & CTTTCAGATGCTACCCT & 0.432 & 0.356 \\
\hline SNP_22686 & $\mathrm{C} / \mathrm{A}$ & TTTCATCATGGGTG & AGACAGGGCAAAAGAA & 0.494 & 0.420 \\
\hline SNP_22699 & $\mathrm{T} / \mathrm{C}$ & AAGGCG & TGCGCCAGCT & 0.175 & 0.148 \\
\hline SNP_22752 & & GTCAGGTTGAGAGGAATTT & TCGATT & & 0.000 \\
\hline SNP_22796 & $\mathrm{C} / \mathrm{T}$ & CGTAATTTATCAGGCCTTCC & GATGGCAGATAC & 0.123 & 0.111 \\
\hline SNP_22990 & $\mathrm{C} / \mathrm{T}$ & AGCAGAATTAATGGCTGCCC & AACGATGGCGTGATA & & 0.346 \\
\hline SNP_23047 & $\mathrm{G} / \mathrm{A}$ & TAAAATTGTCCAGCATCGGC & GGAGCTTTAGGATGGGTTAG & & 0.411 \\
\hline SNP_23189 & $\mathrm{G} / \mathrm{A}$ & CCCAAGGGTTGTAACTCTTC & ATTACGAGCGAAGGACACAG & 0.000 & 0.226 \\
\hline SNP_23198 & $\mathrm{G} / \mathrm{A}$ & CGTGTTGCAGAAGCTTTTCG & GACCCACAAAATGTCAAAGC & 0.432 & 0.396 \\
\hline SNP_23219 & $\mathrm{G} / \mathrm{T}$ & CTGTATGCTCAGTATCCTCC & CGAAAGAGACAACTTTTCGC & 0.148 & 0.317 \\
\hline SNP_23318 & $\mathrm{G} / \mathrm{A}$ & AGCCCACGATACAAAAGCTG & GACGCCTTCATGGTCGAAAT & 0.402 & 0.463 \\
\hline SNP_23352 & $\mathrm{G} / \mathrm{T}$ & GAACCAGCTAACGTTAACGG & AAATCCAGAACACCGCTCAG & 0.404 & 0.423 \\
\hline SNP_23390 & $\mathrm{A} / \mathrm{T}$ & CATGTCAGCCAAAATTAAGG & TGGCAGAGATTGGTTCAAGC & 0.011 & 0.284 \\
\hline SNP_23478 & $\mathrm{C} / \mathrm{T}$ & CATCGAGGCGAGACATAAAG & CTCCGCCAATGAGATTGTTC & 0.127 & 0.139 \\
\hline SNP_23488 & $\mathrm{G} / \mathrm{A}$ & AGACAATCTGTCAAGCTGCG & & 0.000 & 0.000 \\
\hline SNP_2351 & $\mathrm{G} / \mathrm{A}$ & CCTCGTGAATATTGTACCCC & GGGTAACTTTGGCATCAGGG & 0.101 & 0.234 \\
\hline SNP_23693 & $\mathrm{G} / \mathrm{A}$ & CTGGACTTCCTTGGTTTGTG & GAGTGATCGTATGTCTGCTG & 0.383 & 0.383 \\
\hline SNP_23709 & $\mathrm{T} / \mathrm{C}$ & TGAGGGATCGATTTCGGAAC & TCATTTCACGCGTGCGCAAC & 0.026 & 0.045 \\
\hline SNP_23723 & $\mathrm{A} / \mathrm{G}$ & CTGTAAAACACACAGCCAGC & TGTTGTGACTCGGAGTGAAC & 0.400 & 0.361 \\
\hline SNP_23794 & $\mathrm{G} / \mathrm{A}$ & TGAAAGTGCCAGTGAATGTC & CAGCTGCATATAAGCGAAGG & 0.382 & 0.454 \\
\hline
\end{tabular}




\begin{tabular}{|c|c|c|c|c|c|}
\hline SNP ID & Alleles & Primer F & Primer R & Ho & $\mathrm{He}$ \\
\hline SNP_23796 & $\mathrm{G} / \mathrm{A}$ & TCAAAAACGTCTGGTTACCC & ATGCCGACTGTATGTGTTGC & 0.218 & 0.193 \\
\hline & & GTAATCCTTCAAGTACAACTG & & & 0.252 \\
\hline SNP_23817 & $\mathrm{G} / \mathrm{A}$ & CACAAGTTCCTAATCCTATC & CCACCAGCCAGGATATTTTG & 0.240 & 0.318 \\
\hline SNP_23887 & $\mathrm{G} / \mathrm{C}$ & TCCTTGAGACGATGAACTAC & TGATGGTCGATACCATGGG & 0.324 & 0.396 \\
\hline SNP_24069 & $\mathrm{C} / \mathrm{A}$ & AGTGTGTACTACCTGCCAAG & CCTTTGCCATGATTTGGAGG & 0.460 & 0.448 \\
\hline SNP_24148 & $\mathrm{A} / \mathrm{T}$ & TGACATCCTAGACACAGCAG & AAGAATCCTTCGCCACTACG & 0.301 & 0.274 \\
\hline SNP_24220 & $\mathrm{G} / \mathrm{T}$ & AGGCACCATTTGGTCAATCG & GTGTTCTTGAGTTCGTTCTG & 0.272 & 0.317 \\
\hline SNP_24236 & $\mathrm{T} / \mathrm{C}$ & GAGAAACGTACACAGCTCAC & TGTGGAATACTCTGGGTGTC & 0.459 & 0.442 \\
\hline SNP_24295 & $\mathrm{C} / \mathrm{A}$ & ATTGCGTACGCAATCTGGTC & TCTGAGACGTCTGTTGGTTC & 0.366 & 0.337 \\
\hline SNP_2436 & $\mathrm{G} / \mathrm{A}$ & GTGATGTCACCATTTCCTCC & GACAACGCTAAAATCCGACC & 0.321 & 0.325 \\
\hline SNP_24363 & $\mathrm{G} / \mathrm{A}$ & TCGCTTCACTCACAGTTGAC & GCCATTGGCTGATTGCATTG & 0.354 & 0.427 \\
\hline SNP_24376 & $\mathrm{G} / \mathrm{T}$ & CGATCAATTTCATGATCAC & AGAGCGAAACGAGAAC & 0.203 & 0.171 \\
\hline SNP_24405 & $\mathrm{T} / \mathrm{C}$ & TTGQ & GGTTTATAATCTGAACGAGAC & 0.276 & 0.366 \\
\hline SNP_24424 & $\mathrm{A} / \mathrm{G}$ & GGTATTTGTGGATCGTGTGC & GATGTATCATCAATAC & 0.232 & 0.244 \\
\hline SNP_24577 & $\mathrm{T} / \mathrm{C}$ & TGAGGCTAGCGTAGAGAATG & GTTCTCCGTTCTTCTGTTGC & 0.299 & 0.293 \\
\hline SNP_24681 & $\mathrm{T} / \mathrm{C}$ & GGATCTTGTGCGTTGTTTCC & GTGTGAACAACACGCGAGAC & 0.030 & 0.028 \\
\hline SNP_24682 & $\mathrm{T} / \mathrm{A}$ & TTTTTCCAAAGCAGCGCACG & GTGTGACCATTTGAGGT & 0.512 & 0.416 \\
\hline SNP_24774 & $\mathrm{G} / \mathrm{A}$ & CAAGAGGTGTTGAAAACTGC & TGAGCTTC & 0.302 & 0.259 \\
\hline SNP_24818 & $\mathrm{G} / \mathrm{T}$ & CCGTCTGTGATGGAGATAAC & CTGCCT & 68 & 0.275 \\
\hline SNP_24849 & $\mathrm{T} / \mathrm{C}$ & ACTTCCTTTGCACGTCTTCC & TCATGTTATCCAC & & \\
\hline SNP_24998 & $\mathrm{G} / \mathrm{A}$ & AATGAGGTTGTTCACTTCGC & CAATTTCAAGACGATCAGGG & 0.211 & 0.355 \\
\hline & $\mathrm{G} / \mathrm{A}$ & & & & \\
\hline SNP_25227 & $\mathrm{A} / \mathrm{G}$ & GGTTC & GAACGTA & 0.198 & 0.335 \\
\hline SNP_2687 & $\mathrm{G} / \mathrm{A}$ & AATGCTC & CTATAAAC & 0.007 & 0.007 \\
\hline SNP_2764 & $\mathrm{C} / \mathrm{T}$ & TACGGCCAGTACTGTACAAC & CCTTGCCAGTGTTGATCTTG & 0.061 & 0.065 \\
\hline SNP_281 & $\mathrm{G} / \mathrm{C}$ & TGCAAGTAATATTTCGGCAG & TCGAAAAAGA & 0.314 & 0.271 \\
\hline SNP_2969 & $\mathrm{C} / \mathrm{T}$ & GCACTTCCACCAAGAGATAC & ACGCAGGCA & 0.267 & 0.361 \\
\hline SNP_2972 & $\mathrm{G} / \mathrm{A}$ & AGA & TTCAGA & 0.249 & 0.382 \\
\hline & & CGAACT & TC & & 0.077 \\
\hline SNP_3144 & $\mathrm{G} / \mathrm{A}$ & CTTCATTTGGTTCATC & GGCTTA & 0.306 & 0.312 \\
\hline & $\mathrm{A} / \mathrm{G}$ & & & & 0.362 \\
\hline & $\mathrm{C} / \mathrm{T}$ & AAGATCGTGACAACGATGCC & & 0.3 & 0.403 \\
\hline SNP_3217 & $\mathrm{T} / \mathrm{A}$ & AGGTGTAATATCCACGCGTC & ACAGATCCTCTGGTTGCCAC & 0.125 & 0.063 \\
\hline SNP_3297 & $\mathrm{A} / \mathrm{T}$ & TTCATAGCAACACCACGGAC & CCAATGTTGAAAGCTGGTCG & 0.305 & 0.288 \\
\hline SNP_3398 & $\mathrm{A} / \mathrm{T}$ & CGGCTTTTCCTTTGGTTTCG & CAAGGAGGATGAAAACAAGG & 0.272 & 0.269 \\
\hline SNP_372 & $\mathrm{G} / \mathrm{A}$ & GAAAGGATATAGCAAAACACG & CAAATGTCCTTTGGACGCTC & 0.094 & 0.277 \\
\hline SNP_3894 & $\mathrm{G} / \mathrm{A}$ & AGAATTACCTGCTGAGGGAG & TCACGTGCTTCTTCGCATTG & 0.000 & 0.000 \\
\hline SNP_3959 & $\mathrm{G} / \mathrm{C}$ & TCAACTGGCGATCACAGAAC & GGCAGAAACCCGAGAAAATG & 0.150 & 0.201 \\
\hline SNP_4056 & $\mathrm{C} / \mathrm{T}$ & CCCAAGCTAAGTACGCATTG & AGTCGACTCGCCATCTTATG & 0.147 & 0.153 \\
\hline & $\mathrm{T} / \mathrm{A}$ & & TCACCTTCTGCATTGTAACG & 0.113 & 0.109 \\
\hline SNP_4122 & $\mathrm{A} / \mathrm{T}$ & AAACACGGCTTGCCAATGAG & GTCGTTTTGTCAACACCTCC & 0.213 & 0.222 \\
\hline SNP_4165 & $\mathrm{G} / \mathrm{T}$ & АCTTCATTTTCTCCGCGAAC & AACTCCGCCTCAGGATAAAG & 0.470 & 0.453 \\
\hline SNP_4295 & $\mathrm{C} / \mathrm{T}$ & AGCTCCTGTTTCCTTTTGTC & AGAGTCTTCTTCCTTCTCGC & 0.025 & 0.023 \\
\hline SNP_4365 & $\mathrm{A} / \mathrm{G}$ & GAGAGAGTGGTCATTTCGAG & GCATGCTCATCTTCACGTTG & 0.000 & 0.000 \\
\hline SNP 4510 & $\mathrm{~T} / \mathrm{C}$ & TTCGTACCAAGTAATCCTGC & AGGTCTTGGCATCGTCTGTG & 0.869 & 0.479 \\
\hline
\end{tabular}




\begin{tabular}{|c|c|c|c|c|c|}
\hline SNP ID & Alleles & Primer F & Primer R & Ho & $\mathrm{He}$ \\
\hline SNP_4525 & $\mathrm{G} / \mathrm{A}$ & TTCAAACAGCACATCAGCAC & GGTTGAGTTGGCCAAAATCC & 0.350 & 0.338 \\
\hline & & TACAAACACCAGAAGCTTTC & GGGAATGAGAAGACAGAAC & & \\
\hline SNP_4552 & $\mathrm{C} / \mathrm{A}$ & CAGGTGGATGTGTTTCAACG & CGGAAGCCATTCTGTTGAAG & 0.233 & 0.325 \\
\hline SNP_4564 & $\mathrm{C} / \mathrm{T}$ & CTGCTCATATCTGCGTCAAG & TCCAAGTACCTGACTTCTC & 0.000 & 0.063 \\
\hline SNP_4603 & $\mathrm{A} / \mathrm{T}$ & TGGTAAATATTCAACGCAGG & GTTGTACAGGCTTGTCGTTC & 0.132 & 0.220 \\
\hline SNP_4631 & $\mathrm{G} / \mathrm{A}$ & AAGAAACGCCGGGCTATCAG & TTTGCAACGATTCGTCGTCC & 0.139 & 0.150 \\
\hline SNP_4660 & $\mathrm{G} / \mathrm{A}$ & GCGAAAACGTTCACCGTCAA & CTGGCTTTCCAGAAATCTTC & 0.000 & 0.000 \\
\hline SNP_4717 & $\mathrm{G} / \mathrm{A}$ & CCACTTGGCAAGATGATAGG & AAGATGTTTTCAGTGCCGCC & 0.137 & 0.147 \\
\hline SNP_473 & $\mathrm{C} / \mathrm{T}$ & СCСACTACCATAGTATCTCC & TGAGGCATCCCTCCTTTTTG & 0.212 & 0.202 \\
\hline SNP_4871 & $\mathrm{C} / \mathrm{T}$ & CTCTTGTGGAATCACTGACC & CCTTCACTTGTGACATGCTG & 0.293 & 0.223 \\
\hline SNP_5076 & $\mathrm{A} / \mathrm{G}$ & TTCCTGAGCACCTTTGAAGC & TATCGATGCGCTCACTCCAC & 0.295 & 0.263 \\
\hline SNP_5097 & $\mathrm{C} / \mathrm{T}$ & TGTCGAAATCTATGGCTGGG & AATTGGAGGAGATCTTCCAC & 0.000 & 0.174 \\
\hline & $\mathrm{C} / \mathrm{T}$ & AACGATCTGCAACATGGGAG & TTCAGATAG & 0.357 & 0.341 \\
\hline SNP_5278 & $\mathrm{G} / \mathrm{C}$ & TAGGCACGGTGTTTTGTGAG & AAGCGCCTT & 0.404 & 0.442 \\
\hline SNP_5299 & $\mathrm{A} / \mathrm{G}$ & CAACGTGAACGTGTCCTTAG & TTCGAGAGCGCGGATTGAAC & 0.468 & 0.443 \\
\hline SNP_5313 & $\mathrm{G} / \mathrm{T}$ & ATCAGATGTCAGACAGGACG & TTCCAAGAGGAACAAGTGGC & 0.021 & 0.271 \\
\hline SNP_5355 & $\mathrm{C} / \mathrm{T}$ & GAAGTACCTAAGGGACATGC & GCTTCCTCGTCGCTTAATTC & 0.480 & 0.457 \\
\hline SNP_536 & $\mathrm{C} / \mathrm{A}$ & CAAACTTGCGTCACGACATC & CTCTTGAGGACTGCTTGAAC & 0.475 & 0.378 \\
\hline SNP_5397 & $\mathrm{G} / \mathrm{A}$ & AACTTCATCGCATCACGGAC & GTTCTTGCCTGGTGGTTTAG & 0.000 & 0.000 \\
\hline SNP_5421 & $\mathrm{A} / \mathrm{T}$ & AATAGAAGGAGACCAAGGCG & AGTATTCCTCTTGCTCAGCG & 0.000 & 0.094 \\
\hline SNP_5528 & $\mathrm{T} / \mathrm{A}$ & TTACGATGCCGAACTCGTTG & AAATAAAAATCCCGGGAGGC & 0.107 & 0.156 \\
\hline & & & & & 0.433 \\
\hline SNP_5581 & $\mathrm{G} / \mathrm{T}$ & GTTCGGAA & GTTC & 0.321 & 0.283 \\
\hline SNP_5625 & $\mathrm{T} / \mathrm{C}$ & TGTTTGATTCA & TATGCAAGA & 0.235 & 0.240 \\
\hline SNP_5644 & $\mathrm{C} / \mathrm{G}$ & AACACGCGGGATATAGTAGG & TTGCCTAACCACGTGTTTCG & 0.124 & 0.130 \\
\hline SNP_5678 & $\mathrm{C} / \mathrm{T}$ & CAGGAAAGAGATACAGTGAC & CGGTCCAT & 0.418 & 0.334 \\
\hline SNP_5714 & $\mathrm{C} / \mathrm{T}$ & GCACCAGAACACGTAATTCC & CGGGATCAAGGATAAACAGG & 0.066 & 0.063 \\
\hline SNP_5803 & $\mathrm{C} / \mathrm{T}$ & ATTATAGTTC & ACGACACCAC & 0.198 & 0.191 \\
\hline & & CTCGGAACTTGAAGAGATCA & GTCTTAGCGGTTCT & & 0.149 \\
\hline SNP_5899 & $\mathrm{G} / \mathrm{A}$ & CACAACAACGACTTTCGTGC & AGAGTCTGCCTACCACAAAG & 0.000 & 0.000 \\
\hline & $\mathrm{T} / \mathrm{A}$ & TTCTCTTGGTCCTGGAGAAC & ACATAAACAGAGAGAAG & & 0.057 \\
\hline & $\mathrm{A} / \mathrm{G}$ & & & 0.4 & 0.419 \\
\hline SNP_6245 & $\mathrm{G} / \mathrm{T}$ & CTGCAAGGCTTCCATAAAAG & TGACCAAGGCTGATCTTGTC & 0.284 & 0.362 \\
\hline SNP_6308 & $\mathrm{G} / \mathrm{A}$ & TCCCTTGTCAGTAAAGCTGG & CGTTCTTGTTCGTCTTTTCG & 0.396 & 0.386 \\
\hline SNP_6476 & $\mathrm{C} / \mathrm{T}$ & AAAATCAGCACTGCTTAGCG & TGTATGCCAACGACAAGCTG & 0.044 & 0.041 \\
\hline SNP_6505 & $\mathrm{C} / \mathrm{A}$ & CCTCGTCAAGAAGTGTATGG & GGAGAACTGCTCATAGAAGG & 0.313 & 0.284 \\
\hline SNP_6509 & $\mathrm{G} / \mathrm{A}$ & GTTAATGAATCAAAAATGGC & АCTCCCAATGACTCTGCAAC & 0.180 & 0.352 \\
\hline SNP_6711 & $\mathrm{C} / \mathrm{T}$ & GAACTCATCTCGAATGCGTC & CAATCGTCGAGTTTTGAAGG & 0.426 & 0.407 \\
\hline SNP_6751 & $\mathrm{G} / \mathrm{A}$ & GAGTTTGTGAGAATTGATCC & CACATCAGGGAGTTTAAATTG & 0.408 & 0.377 \\
\hline & $\mathrm{T} / \mathrm{C}$ & CATTCACAGGCTTGCTTCTC & & 0.289 & 0.255 \\
\hline SNP_6963 & $\mathrm{A} / \mathrm{T}$ & GTACATGGAAACGTGAGAGC & GGCTGTTCGTTACGATATCT & 0.185 & 0.374 \\
\hline SNP_7006 & $\mathrm{G} / \mathrm{A}$ & GAAATTCTGAAACCAGGCCG & GGTATCAAGTATGACCCAAG & 1.000 & 0.500 \\
\hline SNP_7201 & $\mathrm{C} / \mathrm{T}$ & AGAATTGCAGAGAATGCGAC & GTCGTCCTTCAATACACTTC & 0.400 & 0.350 \\
\hline SNP_7263 & $\mathrm{G} / \mathrm{A}$ & TTCAGAAGGTGAAGTGGTCG & CTAACAGCCATTTCTCGACC & 0.246 & 0.201 \\
\hline SNP 7387 & $\mathrm{C} / \mathrm{T}$ & TCTGGGAATGTCTAACACGC & ATGGGTAACACACGCGATTC & 0.324 & 0.288 \\
\hline
\end{tabular}




\begin{tabular}{|c|c|c|c|c|c|}
\hline SNP ID & Alleles & Primer F & Primer R & Ho & He \\
\hline SNP_7482 & $\mathrm{C} / \mathrm{A}$ & CGGCTCTAACGTGATTTCAG & TCATTACTCCATCTTTGCCG & 0.428 & 0.402 \\
\hline SNP_7541 & $\mathrm{T} / \mathrm{C}$ & & & .028 & 0.026 \\
\hline SNP_7562 & $\mathrm{C} / \mathrm{T}$ & TATGCACTTCCTCGGGTAAC & TCAAGCTACCACAGATGAG & & \\
\hline SNP_7579 & G/ A & СТCTCTCATTTTCTTGCCTC & & 0.349 & 0.396 \\
\hline SNP_7614 & $\mathrm{C} / \mathrm{A}$ & TCACTTATCACCTGGTCGAG & GAAGATTATCTCTGAAGCGG & 0.280 & 0.235 \\
\hline SNP_7619 & $\mathrm{T} / \mathrm{C}$ & AACAGTACAAGGTCGTTGGG & GGTGCAAAGATGTTCATACG & 0.352 & 0.261 \\
\hline & $\mathrm{T} / \mathrm{C}$ & AAATGCAGCTCGACATCGAC & TAGTAATCCCAGCCCATGTC & 0.339 & 0.334 \\
\hline SNP_7739 & $\mathrm{C} / \mathrm{G}$ & TAAGACTGCATCAACCGCTG & ACAACACG & 0.319 & 0.308 \\
\hline SNP_7760 & $\mathrm{G} / \mathrm{T}$ & ACTCCACAATATCGCTGCAC & TGAAAAGCCAACGCCCAAG & 279 & 0.221 \\
\hline SNP_7783 & $\mathrm{T} / \mathrm{C}$ & TGACGATTCCAGCTTCACAG & GGAGTGGTTCTTTTGGAGAC & 0.000 & 0.000 \\
\hline SNP_7893 & $\mathrm{G} / \mathrm{C}$ & & & & 0.380 \\
\hline SNP_7976 & $\mathrm{T} / \mathrm{C}$ & ATCTCAGGCTGAGGAAGATG & GTTCTGAATGGCTCTACCAC & 0.141 & 0.120 \\
\hline SNP_799 & $\mathrm{C} / \mathrm{T}$ & AATGCTCTCGTACCTTCTAC & & & 0.000 \\
\hline SNP_8002 & $\mathrm{C} / \mathrm{T}$ & GCA & & 83 & 0.223 \\
\hline SNP_8041 & $\mathrm{A} / \mathrm{T}$ & ATGTTCACAAGATTGGCCTC & СТТТССТССТССАССА & 96 & 0.428 \\
\hline SNP_8129 & $\mathrm{C} / \mathrm{A}$ & CCACTACACTCGACATCATC & & 865 & 0.482 \\
\hline SNP_8159 & G/ A & TCCGTCATGTAGAGAACCTC & GAGTCCAAAGATCAAC & 0.000 & 0.000 \\
\hline SNP_8219 & $\mathrm{C} / \mathrm{T}$ & TGATGTCAGCAGTTGATGCC & CCGTTTGTATGAGCTT & 0.300 & 0.405 \\
\hline SNP_8320 & $\mathrm{T} / \mathrm{C}$ & GGGTCGTTGACAATTTCATC & AAATCAGAAGACGTT & 0.304 & 0.301 \\
\hline SNP_8327 & $\mathrm{C} / \mathrm{T}$ & GGCAGTGACAATTTCTC & & 0.161 & 0.155 \\
\hline SNP_8381 & $\mathrm{G} / \mathrm{A}$ & CATGCTCCTCATCATTCTCC & TGTTCAGCGGTCAGGTTAAG & 0.154 & 0.144 \\
\hline SNP_8421 & $\mathrm{T} / \mathrm{C}$ & CTTGGTGATATTCTCATGGG & TACACAGGGTACAGATAGGG & 0.025 & 0.024 \\
\hline SNP_8440 & $\mathrm{G} / \mathrm{A}$ & AAGTTGCCAGAGAGCGAAAG & TAAGTTCAACATCGCAT & 0.415 & 0.324 \\
\hline SNP_8543 & $\mathrm{A} / \mathrm{G}$ & TGCTCTTAACTTCTGGTCTG & CCAAGCTGTTTCGTGATTTG & 0.338 & 0.356 \\
\hline & $\mathrm{C} / \mathrm{T}$ & CCAGAACTTTGGCGTGCTTG & GATCATAAGCACGTGATTGG & & 0.372 \\
\hline SNP_881 & $\mathrm{C} / \mathrm{T}$ & AGTAGTTCATCGTCTCAAGG & GACAGGTCTCCATATCTGTG & 0.362 & 0.315 \\
\hline SNP_9014 & $\mathrm{C} / \mathrm{T}$ & GACGTTTTTGGTTGCGTCTG & AAAGTTCCTGTGGTTGGTGG & 0.269 & 0.243 \\
\hline SNP_9146 & $\mathrm{C} / \mathrm{G}$ & GGGCTACACTATTTCATTGC & AACAGCCCTATTTCGATGAC & 0.244 & 0.291 \\
\hline SNP_9347 & $\mathrm{T} / \mathrm{C}$ & CATCAAAGAAGTGCTTGCTG & GGCAATAAGTCAGGCAGAAG & 0.429 & 0.391 \\
\hline SNP_9393 & $\mathrm{C} / \mathrm{T}$ & TGGTGGTTACCCAATTGCTG & CCTTGGCTACTGTTGTACTG & 0.198 & 0.250 \\
\hline SNP_9431 & $\mathrm{T} / \mathrm{C}$ & TTGCCAAAGATAAAGGCGAC & GTGTTTCCAAAAGGACCTCG & 0.363 & 0.342 \\
\hline SNP_9910 & $\mathrm{T} / \mathrm{C}$ & AATCAGAAGCGCTCTTCACC & CACAGAAGTCGTGAAGTTCG & 0.225 & 0.218 \\
\hline SNP_9933 & $\mathrm{C} / \mathrm{T}$ & GATTGGGACTTTTTGCCTGC & GCATCATCCACCCAATCATC & 0.000 & 0.000 \\
\hline SNP_9998 & $\mathrm{C} / \mathrm{T}$ & CTGCTTCCAAGCCATTTAAG & GAAAATTGCCAATCTCAACGG & 0.000 & 0.000 \\
\hline
\end{tabular}


Table 1.- Location and number of individuals (n) of Paramuricea clavata used for SNP selection using MassArray

\begin{tabular}{|l|l|c|c|l|}
\hline Location & Site & Latitude & Longitude & n \\
\hline \multirow{4}{*}{ Bastia } & Lavasina & 42.7552 & 9.4783 & 10 \\
\cline { 2 - 5 } & Roche Mimosa 1 & 42.7072 & 9.4605 & 10 \\
\cline { 2 - 5 } & Roche Mimosa 2 & 42.7078 & 9.461 & 10 \\
\hline \multirow{3}{*}{ La Spezia } & Tinetto Parete & 44.0236 & 9.8505 & 10 \\
\cline { 2 - 5 } & Tinetto Secca & 44.0226 & 9.8513 & 10 \\
\cline { 2 - 5 } & Punta Tino & 44.0293 & 9.8472 & 10 \\
\hline \multirow{3}{*}{ Punta Mesco } & Punta Mesco 1 & 44.1326 & 9.6345 & 10 \\
\cline { 2 - 5 } & Punta Mesco 2 & 44.1324 & 9.6349 & 10 \\
\hline Toulon & & 43.0438 & 6.0610 & 15 \\
\hline
\end{tabular}

